\title{
THE AUTOMORPHISM GROUP OF A PRODUCT OF GRAPHS
}

\author{
DONALD J. MILLER
}

\begin{abstract}
In a recent paper we showed that every connected graph can be written as a weak cartesian product of a family of indecomposable rooted graphs and that this decomposition is unique to within isomorphisms. Using this unique prime factorization theorem we prove that if a graph $X$ can be written as a product of connected rooted graphs, which are pairwise relatively prime, then the automorphism group of $X$ is isomorphic to the restricted direct product of the automorphism groups of the factors with prescribed subgroups the isotropy groups of the factors at the roots. This is a generalization of Sabidussi's theorem for cartesian multiplication.
\end{abstract}

Sabidussi [4] proved that if $X_{1}, \cdots, X_{n}$ are connected graphs of finite type which are pairwise relatively prime with respect to cartesian multiplication, then the automorphism group of the product is isomorphic to the direct product of the automorphism groups of the factors. The proof of this result uses the unique prime factorization theorem for cartesian multiplication. In [3] it was proved that, for connected graphs, unique prime factorization holds for weak cartesian multiplication. The purpose of this note is to generalize Sabidussi's Theorem to the weak cartesian product of a family of rooted graphs.

Let $\left(G_{a}\right)_{a \in A}$ be a family of groups and for each $a \in A$ let $H_{a}$ be a subgroup of $G_{a}$. The restricted direct product of the family of groups $\left(G_{a}\right)_{a \in A}$ with prescribed subgroups $H_{a}$ is defined by

$$
\prod_{a \in A}\left(G_{a}, H_{a}\right)=\left\{g \in \prod_{a \in A} G_{a} \mid \operatorname{pr}_{a} g \in H_{a} \text { for all but finitely many } a \in A\right\} \text {. }
$$

This product was introduced by Vilenkin [6] and can be found in Kuroš [2]. For a subgraph $Y$ of a graph $X$ we use the notation of [5] and denote the subgroup of the automorphism group of $X$ that leaves $Y$ invariant by $G(X ; Y)$, that is, $G(X ; Y)=\{\phi \in G(X) \mid \phi Y=Y\}$, where $G(X)$ is the automorphism group of $X$. Hence $G\left(X_{a} ; x_{a}\right)$ denotes the isotropy group of $X_{a}$ at $x_{a}$. Our main result is the following:

THEOREM. Let $\left(X_{a}, x_{a}\right)_{a \in A}$ be a family of connected rooted graphs which are pairwise relatively prime. Then $G\left(\prod_{a \in A}\left(X_{a}, x_{a}\right)\right)$ is isomorphic to

Received by the editors July 30, 1969.

A MS Subject Classifications. Primary 0562; Secondary 2022, 2052.

Key Words and Phrases. Automorphism group, weak cartesian product of graphs, cartesian product of graphs, restricted direct product of groups, sum of graphs. 
the restricted direct product of the groups $G\left(X_{a}\right)$ with prescribed subgroups $G\left(X_{a} ; x_{a}\right)$.

We will proceed by first characterizing a particular subgroup of the automorphism group of the disjoint union of a family of connected graphs. Let $\left(X_{a}\right)_{a \in A}$ be a family of connected graphs and for each $a \in A$ we distinguish a vertex $x_{a} \in V\left(X_{a}\right)$, that is, we consider the family of rooted graphs $\left(X_{a}, x_{a}\right)_{a \in A}$. Let $X=\sum_{a \in A}\left(X_{a}, x_{a}\right)$ denote the disjoint union of the family $\left(X_{a}, x_{a}\right)_{a \in A}$. Since each $X_{a}$ is connected, every $\phi \in G(X)$ determines a permutation $\pi_{\phi}$ on the index set $A$ by $\pi_{\phi}(a)=b$ if and only if $\phi X_{a}=X_{b}$. Let $H(X)$ be the subgroup of $G(X)$ consisting of all $\phi \in G(X)$ such that $\phi x_{a}=x_{\pi_{\phi}(a)}$ for all but finitely many $a \in A$.

Lemma 1. Let $\left(X_{a}, x_{a}\right)$ be a family of connected graphs. Let $X=$ $\sum_{a \in A}\left(X_{a}, x_{a}\right)$ and let $N=H(X) \cap \bigcap_{a \in A} G\left(X ; X_{a}\right)$. Then $N$ is normal in $H(X)$ isomorphic to the restricted direct product of $G\left(X_{a}\right)$ with prescribed subgroups $G\left(X_{a} ; x_{a}\right)$ and $H(X) / N$ is isomorphic to a union of an upward directed family of direct products of symmetric groups.

Proof. For any $\phi \in H(X), \psi \in N$ and any $a \in A$,

$$
\phi^{-1} \psi \phi X_{a}=\phi^{-1} \psi X_{\pi_{\phi}(a)}=\phi^{-1} X_{\pi_{\phi}(a)}=X_{a},
$$

that is, $\phi^{-1} \psi \phi \in N$. Hence $N$ is normal in $H(X)$.

To show that $N$ is isomorphic to the restricted product of $G\left(X_{a}\right)$ with prescribed subgroups $H_{a}=G\left(X_{a} ; x_{a}\right)$, let $P=\prod_{a \in A}\left(G\left(X_{a}\right), H_{a}\right)$ and define $f: N \rightarrow P$ by

$$
\operatorname{pr}_{a}(f \phi)=\phi \mid X_{a}, \quad \text { for } \phi \in N .
$$

Clearly $\phi \mid X_{a} \in G\left(X_{a}\right)$ and $\phi \mid X_{a} \in G\left(X_{a} ; x_{a}\right)$ for all but a finite number of $a \in A$, and hence $f \phi \in P$. It is easily verified that $f$ is an isomorphism.

To prove that $H(X) / N$ is isomorphic to a union of direct products of symmetric groups we partition $A$ into a collection of subsets $\left\{A_{i} \mid i \in I\right\}$ such that $X_{a} \cong X_{b}$ if and only if $a, b \in A_{i}$ for some $i \in I$. Now partition $A_{i}$ into a collection of subsets $\left\{A_{i j} \mid j \in J_{i}\right\}$ such that $a, b \in A_{i j}$ for some $j \in J_{i}$ if and only if there exists an isomorphism $\phi$ : $X_{a} \rightarrow X_{b}$ such that $\phi x_{a}=x_{b}$. We will assume that the index sets $J_{i}$ are chosen to be disjoint. We will denote the symmetric group on a set $A$ by $S(A)$ and if $B \subset A$ we will consider $S(B) \subset S(A)$. For any finite subset $K$ of $\bigcup_{i \in I} J_{i}$ put $K_{i}=K \cap J_{i}$ and let

$$
P_{K_{i}}=S\left(\bigcup_{j \in K_{i}} A_{i j}\right) \times \prod_{j \in J_{i}-K_{i}} S\left(A_{i j}\right)
$$

and 


$$
P_{K}=\prod_{i \in I} P_{K_{i}}
$$

If $K$ and $K^{\prime}$ are any two finite subsets of $\bigcup_{i \in I} J_{i}$ then $P_{K}$ and $P_{K^{\prime}}$ are subgroups of $P_{K \cup K^{\prime}}$, that is, the $P_{K}$ 's form an upward directed family of subgroups of $S(A)$ and hence $U P_{K}$, where the union is taken over all finite subsets of $\bigcup_{i \in I} J_{i}$, is a group. The homomorphism $\phi \rightarrow \pi_{\phi}$ of $H(X)$ into $S(A)$ has as its kernel $N$. We will now show that $\phi \rightarrow \pi_{\phi}$ is a mapping onto $U P_{K}$. For any $\phi \in H(X)$ we have that

$$
X_{\pi_{\phi}(a)}=\phi X_{a} \cong X_{a}
$$

and hence $\pi_{\phi} \mid A_{i} \in S\left(A_{i}\right)$. Now $\phi \in H(X)$ implies that $\phi x_{a}=x_{\pi_{\phi}(a)}$ for all but a finite number of $a \in A$ and hence $\pi_{\phi} \mid A_{i j} \in S\left(A_{i j}\right)$ for all but a finite number of $j \in J_{i}$. Let $K$ be the finite subset of $\bigcup_{i \in I} J_{i}$ such that $j \in K$ if and only if $\pi_{\phi} \mid A_{i j} \in S\left(A_{i j}\right)$ and let $K_{i}=K \cap J_{i}$. Then for each $i \in I, \pi_{\phi} \mid A_{i} \in P_{K_{i}}$ and therefore

$$
\pi_{\phi} \in \prod_{i \in I} P_{K_{i}}=P_{K}
$$

Now for $a, b \in A_{i j}$ let $\tau_{a, b}: X_{a} \rightarrow X_{b}$ be an isomorphism such that $\tau_{a, b}\left(x_{a}\right)=x_{b}$ and for any $a, b \in A_{i}$ let $\sigma_{a, b}: X_{a} \rightarrow X_{b}$ be an isomorphism. Let

$$
\pi \in \cup P_{K}
$$

and let $K_{0}$ be any finite subset of $\bigcup_{i \in I} J_{i}$ such that $\pi \in P_{K_{0}}$. Define $\phi: X \rightarrow X$ by

$$
\begin{aligned}
& \phi \mid X_{a}=\tau_{a, \pi(a)}, \quad \text { if } a \notin K_{0} \text {, } \\
& =\sigma_{a, \pi(a)}, \text { if } a \in K_{0} .
\end{aligned}
$$

Clearly $\pi_{\phi}=\pi$ and hence $H(X) / N$ is isomorphic to $U P_{K}$.

Corollary 1. If $\left(X_{a}, x_{a}\right)_{a \in A}$ is a family of connected nonisomorphic graphs then

$$
H\left(\sum_{a \in A}\left(X_{a}, x_{a}\right)\right) \cong \prod_{a \in A}\left(G\left(X_{a}\right), G\left(X_{a} ; x_{a}\right)\right) .
$$

Proof. Follows since $G\left(X ; X_{a}\right)=G(X)$ for each $a \in A$, and hence $H\left(\sum_{a \in A}\left(X_{a}, x_{a}\right)\right)=N$.

The following lemma is a generalization of $[4,(3.1)] .{ }^{1}$ 
Lemma 2. Let $\left(X_{a}, x_{a}\right)_{a \in A}$ be a family of connected graphs that are pairwise relatively prime with respect to weak cartesian multiplication. Then

$$
H\left(\sum_{a \in A}\left(X_{a}, x_{a}\right)\right)=G\left(\prod_{a \in A}\left(X_{a}, x_{a}\right)\right) .
$$

Proof. Let $S=\sum_{a \in A}\left(X_{a}, x_{a}\right)$ and $P=\prod_{a \in A}\left(X_{a}, x_{a}\right)$. Define a mapping $f: H(S) \rightarrow G(P)$ by

$$
\operatorname{pr}_{\pi \psi(a)}(f \psi) x=\psi \operatorname{pr}_{a} x, \quad \psi \in H(S),
$$

where $\operatorname{pr}_{a}$ denotes the $a$ th projection of $P$ onto $X_{a}$. Since $\psi x_{a}=x_{x_{\psi}(a)}$ for all but a finite number of $a \in A$ we have that $f \psi: V(P) \rightarrow V(P)$. The proof of $[4,(3.1)]$ then establishes that $f \psi$ is a graph automorphism and that $f$ is a group monomorphism. We now will show that $f$ is onto.

Let $\sigma$ be that equivalence defined on the edge set $E(P)$ by $e \sigma e^{\prime}$ if and only if there exists an $a$ such that the $a$ th projections of $e$ and $e^{\prime}$ are edges of $E\left(X_{a}\right)$. By [3, Remark 2] $\sigma$ is contained in the principal filter of all acyclic equivalence relations containing $\alpha \cup \beta$. By [3, Theorem 2] the least element $\rho$ in the filter of all acyclic equivalences containing $\alpha \cup \beta$ determines the unique decomposition of $P$ into its prime factorization say $P \cong \prod_{b \in B}\left(Y_{b}, y_{b}\right)$. For convenience we will identify $P$ with $\prod_{b \in B}\left(Y_{b}, y_{b}\right)$ and hence we may without loss of generality assume that for each $a \in A$ there exists $C \subset B$ such that $X_{a}=\prod_{b \in C}\left(Y_{b}, y_{b}\right)$ and $\mathrm{pr}_{b} x_{a}=y_{b}$ for all $b \in C$.

Now let $\phi \in G(P)$. For any $z \in V(P)$ and any $a \in A$ let $i_{a}^{z}: X_{a} \rightarrow P$ denote the injection mapping $[4,(2.4)]$ or $[3$, Definition 6$]$ and let $X_{a}^{z}=i_{a}^{2} X_{a}$. Since $\rho$ is invariant under automorphisms this implies that $\phi$ determines a permutation on $B$ by

$$
\phi Y_{b}^{x}=Y_{\pi_{\phi}(b)}^{\phi(x)}, \quad b \in B,
$$

where $x \in V(P)$ is defined by $\operatorname{pr}_{a} x=x_{a}, a \in A$. Since the $X_{a}$ 's are relatively prime and since $\rho \subset \sigma(1)$ then implies that $\rho$ also determines a permutation on $A$ given by

$$
\phi X_{a}^{x}=X_{\pi_{\phi}(a)}^{\phi(x)}, \quad a \in A .
$$

Now define $\psi \in H(S)$ by

$$
\psi \mid X_{a}=\operatorname{pr}_{\pi_{\phi}(a)} \phi i_{a}^{x}, \quad a \in A .
$$

Again using the proof of $[4,(3.1)]$ one can show that $f \psi=\phi$. Hence $f$ 
is onto and therefore an isomorphism. This completes the proof of Lemma 2.

The proof of the theorem now follows from Corollary 1 and Lemma 2.

\section{REFERENCES}

1. W. Imrich, Über das schwache kartesische Product von Graphen, J. Combinatorial Theory (to appear).

2. A. Kuroš, Theory of groups. Vol. I, 2nd ed., GITTL, Moscow, 1953; English transl., Chelsea, New York, 1960. MR 15, 501; MR 22 \#727.

3. D. J. Miller, Weak Cartesian product of graphs, Colloq. Math. (to appear).

4. G. Sabidussi, Graph multiplication, Math. Z. 72 (1959/60), 446-457. MR 35 $\# 80$.

5. — Vertex-transitive graphs, Monatsh. Math. 68 (1964), 426-438. MR 31 \#91.

6. N. Ja. Vilenkin, Direct decompositions of topological groups. I, Mat. Sb. 19 (61) (1946), 85-154; English transl., Amer. Math. Soc. Transl. (1) 8 (1962), 78-154. MR 8, 132.

University of Victoria, Victoria, British Columbia, Canada

${ }^{1}$ In [1, Satz 7], Imrich has an elementwise description of $G\left(\Pi_{a \in A}\left(X_{a}, x_{a}\right)\right)$ which is essentially the same as our Lemma 2 with prime replacing pairwise relatively prime. 